\title{
Adaptation of the control synthesized by the ACAR method to the control based on the implementation of the maximum principle
}

\author{
Viktor P. Lapshin ${ }^{1}$, Ilya A. Turkin ${ }^{1}$, Alexey A. Zakalyuzhnyy ${ }^{1, *}$, Viktor F. Khlystunov ${ }^{2}$, and \\ Gennadiy A. Kuzin ${ }^{1}$ \\ ${ }^{1}$ Don State Technical University, 344000 Rostov-on-Don Gagarin square 1, Russia \\ ${ }^{2}$ State Scientific Establishment "Agriculture research center "Donskoy", North Caucasus Institute of \\ Mechanization and Electrification of Agriculture, 347740 Zernograd, Russia
}

\begin{abstract}
A special case of synthesizing the electromechanical control system by the maximum method and using the Analytical Construction method of Aggregate Regulators (ACAR) is considered in the article. For the basis the task of synthesizing the optimal for speed electromechanical positioning system was chosen, while the moment of resistance to movement linearly depended on the output coordinate of the system, that is, on the angle of the engine rotor rotation. Synthesis of the optimal system for speed makes it possible to increase the efficiency of the entire production process in many production tasks, and the synthesis of the optimal linear control system based on the maximum principle is a fairly well-formalized problem. Here it should be noted that the procedure for synergistic synthesis of the optimal control system has no such formalization. An approach that brings together the solutions obtained by these two methods, which makes it possible to increase the efficiency of the ACAR method by adding some features of the methodology for synthesizing optimal systems by introducing nonlinearity of the "saturation" type is proposed in the article. The results obtained made it possible to formulate the following basic scientific proposition: the synthesis of a control system based on the synergetic approach makes it possible to obtain a system close to optimal (quasi-optimal, but after the modification of the synergetic synthesis method itself.) Here we also formulate the hypothesis of a connection between the time constants, using the ACAR method, with the optimal control switching time determined in the maximum method.
\end{abstract}

\section{Statement of the research task}

In the previous century, the needs of the development of technology, in particular space, became the basis for a new management theory, which can be generalized by the concept of

\footnotetext{
* Corresponding author: zakalizhnuy-95@yandex.ru
} 
optimal control theory [1]. At the heart of this new direction then lies the maximum method developed in the fifties and sixties of the twentieth century, over which Soviet mathematicians L.S. Pontryagin and his disciples worked [1]. In this case, the application of this method is based on the formalization of the synthesis problem with the transition to the form of the Mayer problem and, subsequently, the solution, as a rule, of systems of linear equations [1]. Here it is worth noting that the need to solve systems of differential equations, and in the nonlinear case this is not always possible, is a limitation of the method.

At the end of the 20th century a new approach to the synthesis of control systems appeared, which from its author Kolesnikov AA. was named the method of Analytical Design of Aggregated Regulators (ACAR) [2-3]. This approach is based on the concept of synthesis of nonlinear feedbacks that provide the asymptotic stability of the control system with respect to the required (from the practical point of view) motion (attractor) in the state space of the system [2]. The difference between this method and the methods of synthesis of optimal control systems is that here as a criterion for optimizing the control system is absent. From the point of view of realization of the synthesis procedure, the ACAR method has an undoubted advantage over the methods of synthesis of optimal systems, expressed in the absence of restrictions on the nonlinearity of the initial system of differential equations [1].

From a practical point of view, the synthesis of control of processes or objects should still answer the question whether synthesized management is the property of superiority over all others. This is due to the fact that modern mass production requires increased efficiency. One of the ways to increase this efficiency is to optimize the management processes for speed. This approach is applicable both to systems of automated assembly of equipment [4], and to metal-cutting systems, in particular to drilling control systems in metal-cutting machines [5-8]. From a scientific point of view, it is actual to check the solution obtained by the ACAR method obtained with allowance for the nonlinear dynamics of processes in engineering [5-13], in comparison with the solution obtained on the basis of the maximum method [1].

\section{Basic mathematical model of electromechanical system and synthesis of control based on Pontryagin and ACAR methods}

At the heart of the electromechanical control system in the case under consideration is a direct current motor, which ensures the conversion of electrical energy into mechanical energy of the motion of the system actuators. The mathematical model of a DC electric motor with a collector control is described by the following system of equations [13]:

$$
\begin{aligned}
& U-c_{e} \omega=L \frac{d i}{d t}+R i \\
& c_{m} i=J \frac{d \omega}{d t}+M_{c}
\end{aligned}
$$

where $\mathrm{U}$ is the voltage applied to the engine collector;

$\mathrm{i}$ - current consumed by the engine;

$\mathrm{R}, \mathrm{L}$ - parameters of the engine electrical part;

$\mathrm{J}$ - parameter characterizing inertial properties of the motor rotor, reduced inertial moment of all rotating masses;

$\omega$ is the rotor speed of the engine; 
$M_{c}$ - external, attached moment of resistance;

$c_{m}, c_{e}$ - mechanical and electrical constants of the engine;

In the case under consideration, the moment is represented as a function of the rotation angle of the motor rotor, that is $M_{c}=C \alpha$, taking into account (1) we rewrite:

$$
\begin{aligned}
& U-c_{e} \omega=L \frac{d i}{d t}+R i \\
& c_{m} i=J \frac{d \omega}{d t}+C \alpha \\
& \frac{d \alpha}{d t}=\omega
\end{aligned}
$$

In the second equation we express the value of the current and substitute the value obtained in the first equation, we obtain:

$$
\begin{aligned}
& U \frac{1}{c_{e}}-\omega=\frac{L J}{c_{e} c_{m}} \frac{d^{2} \omega}{d t^{2}}+\frac{R J}{c_{e} c_{m}} \frac{d \omega}{d t}+\frac{L C}{c_{e} c_{m}} \omega+\frac{R C}{c_{e} c_{m}} \alpha \\
& \frac{d \alpha}{d t}=\omega
\end{aligned}
$$

We solve the first equation with respect to the highest derivative and rewrite it with the second, we obtain:

$$
\begin{aligned}
& \frac{d \alpha}{d t}=\omega \\
& \frac{d^{2} \omega}{d t^{2}}=-\frac{R}{L} \frac{d \omega}{d t}-\left(\frac{c_{m} c_{e}}{L J}+\frac{C}{J}\right) \omega-\frac{R C}{L J} \alpha+\frac{c_{m}}{L J} U
\end{aligned}
$$

We denote the variables $\alpha=x_{1}, \omega=x_{2}, \frac{d \omega}{d t}=x_{3}$, as constants, we introduce $\frac{R}{L}=a_{33}$, $\frac{c_{m} c_{e}}{L J}+\frac{C}{J}=a_{32}, \frac{R C}{L J}=a_{31}, \frac{c_{m}}{L J}=b$, then the system (4) takes the form:

$$
\begin{aligned}
& \frac{d x_{1}}{d t}=x_{2} \\
& \frac{d x_{2}}{d t}=x_{3} \\
& \frac{d x_{3}}{d t}=-a_{31} x_{1}-a_{32} x_{2}-a_{33} x_{3}+b U
\end{aligned}
$$

Or in a matrix-vector form:

$$
\dot{\mathbf{x}}=\mathbf{A} x+\mathbf{B} u
$$

where $A=\left(\begin{array}{ccc}0 & 1 & 0 \\ 0 & 0 & 1 \\ -a_{31} & -a_{32} & -a_{33}\end{array}\right)$, 


$$
\begin{aligned}
& B=\left(\begin{array}{l}
0 \\
0 \\
b
\end{array}\right), \\
& x=\left(\begin{array}{l}
x_{1} \\
x_{2} \\
x_{3}
\end{array}\right), \\
& u=\left(\begin{array}{l}
0 \\
0 \\
U
\end{array}\right) .
\end{aligned}
$$

We take the DC motor as the drive providing the motion of the system under consideration, the parameters of which are provided by the following values of the constants: $\mathrm{a}_{31}=4.65, \mathrm{a}_{32}=4.6, \mathrm{a}_{33}=2$. Taking this into account, the matrix A takes the form: $A=\left(\begin{array}{ccc}0 & 1 & 0 \\ 0 & 0 & 1 \\ -4.65 & -4.6 & -2\end{array}\right)$. Knowing the matrix A, we find the eigenvalues $\lambda$ of the matrix A: $\lambda_{1}=-1.2666+0.0000 \mathrm{i}, \lambda_{2}=-0.3667+1.8806 \mathrm{i}, \lambda_{3}=-0.3667-1.8806 \mathrm{i}$.

As can be seen from the obtained eigenvalues of the matrix A, the control system is Lyapunov stable [14]; moreover, one can assert that the processes occurring in the system are vibrational, since the eigenvalues not only contain negative real parts but have nonzero imaginary parts.

The task of synthesizing optimal control will be as follows:

- Using the maximum principle for the object described by the system (5), determine the algorithm of the optimal equation that ensures the transfer of the object from the initial state $\mathrm{x}_{1}(0), \mathrm{x}_{2}(0), \mathrm{x}_{3}(0)$ to the final state $\mathrm{x}_{1}(\mathrm{~T}), \mathrm{x}_{2}(\mathrm{~T}), \mathrm{x}_{3}(\mathrm{~T})$ for the minimum time $\mathrm{T}$, in this case, determine the number of switching operations, the switching times, and construct the control curves $\mathrm{u}(\mathrm{t})$ and the coordinates $\mathrm{x}_{1}(\mathrm{t}), \mathrm{x}_{2}(\mathrm{t}), \mathrm{x}_{3}(\mathrm{t})$. The constraint $|\mathrm{u}| \leq \mathrm{U}$ max is imposed on the control action. System Settings: $\mathrm{a}_{31}=1, \mathrm{a}_{32}=12, \mathrm{a}_{33}=20, \mathrm{~b}=1$; $\mathrm{U}_{\max }=440 \mathrm{~V}$.

At the beginning of the solution of the problem, we define the Hamiltonian

$$
H=\sum_{i=1}^{2} \psi_{i} f_{i}
$$

where $\psi_{i}$ и $f_{i}$ - coordinates of vectors $\boldsymbol{\psi}$ и $\mathbf{f}$.

In addition to the system (5), we formulate a system of equations for the auxiliary variables $\psi \mathrm{i}$, where $\mathrm{i}=1,2$, using the following relationship:

$$
\frac{d \psi_{i}}{d t}=-\sum_{j=1}^{2} \psi_{i} \frac{\partial f_{i}}{\partial x_{i}}, \quad i=1,2
$$

Or in the open form:

$$
\frac{d \psi_{1}}{d t}=-\left[\frac{\partial f_{1}}{\partial x_{1}} \psi_{1}+\frac{\partial f_{2}}{\partial x_{1}} \psi_{2}+\frac{\partial f_{3}}{\partial x_{1}} \psi_{3}\right]
$$




$$
\begin{aligned}
& \frac{d \psi_{2}}{d t}=-\left[\frac{\partial f_{1}}{\partial x_{2}} \psi_{1}+\frac{\partial f_{2}}{\partial x_{2}} \psi_{2}+\frac{\partial f_{3}}{\partial x_{2}} \psi_{3}\right] \\
& \frac{d \psi_{3}}{d t}=-\left[\frac{\partial f_{1}}{\partial x_{3}} \psi_{1}+\frac{\partial f_{2}}{\partial x_{3}} \psi_{2}+\frac{\partial f_{3}}{\partial x_{3}} \psi_{3}\right]
\end{aligned}
$$

Taking into account (5), the system of equation (8) takes the form:

$$
\left\{\begin{array}{l}
\frac{d \psi_{1}}{d t}=4.65 \psi_{3} \\
\frac{d \psi_{2}}{d t}=-\psi_{1}+4.6 \psi_{3} \\
\frac{d \psi_{3}}{d t}=-\psi_{2}+2 \psi_{3}
\end{array}\right.
$$

From the resulting system of equations, we formulate the matrix $A^{\psi}$ :

$$
A^{\psi}=\left(\begin{array}{ccc}
0 & 0 & 4.65 \\
-1 & 0 & 4.6 \\
0 & -1 & 2
\end{array}\right)
$$

The normalized matrix consisting of eigenvectors will take the following form:

$$
V^{\psi}=\left(\begin{array}{ccc}
-0.9474+0.0000 i & 0.5812-0.3347 i & 0.5812+0.3347 i \\
-0.1893+0.0000 i & 0.6883+0.0000 i & 0.6883+0.0000 i \\
-0.2581+0.0000 i & 0.1812+0.2086 i & 0.1812-0.2086 i
\end{array}\right)
$$

Then the diagonalized matrix with eigenvalues:

$$
A^{\psi-D}=\left(\begin{array}{ccc}
1.2666+0.0000 \mathrm{i} & 0 & 0 \\
0 & 0.3667+1.8806 \mathrm{i}, & 0 \\
0 & 0 & 0.3667-1.8806 \mathrm{i}
\end{array}\right)
$$
form:

The solution of the system (9) for the diagonalized case of the matrix $A^{\psi-D}$ takes the

$$
\left\{\begin{array}{l}
\psi_{1}^{*}=C_{1} * e^{1.2666 t} \\
\psi_{2}^{*}=C_{2} * e^{(0.3667+1.8806 i) t} \\
\psi_{3}^{*}=C_{3} * e^{(0.3667-1.8806 i) t}
\end{array}\right.
$$

Taking into account the matrix $V^{\psi}$ in the original basis the solution takes the form:

$$
\left\{\begin{array}{l}
\psi_{1}=-C_{1} * 0.9474 * e^{1.2666 t}+C_{2} *(0.5812-0.3347 i) * e^{(0.3667+1.8806 i) t}+C_{3} *(0.5812+0.3347 i) * e^{(0.3667-1.8806 i) t} \\
\psi_{2}=-C_{1} * 0.1893 * e^{1.2666 t}+C_{2} * 0.6883 * e^{(0.3667+1.8806 i) t}+C_{3}^{*} 0.6883^{*} e^{(0.3667-1.8806 i) t} \\
\psi_{3}=-C_{1} * 0.2581 * e^{1.2666 t}+C_{2} *(0.1812+0.2086 i) * e^{(0.3667+1.8806 i) t}+C_{3} *(0.1812-0.2086 i) * e^{(0.3667-1.8806 i) t}
\end{array}\right.
$$


where $\mathrm{C}_{1}, \mathrm{C}_{2}$, and $\mathrm{C}_{3}$ are integration constants.

A general expression describing the Hamiltonian function:

$$
H=\Psi_{1} f_{1}+\Psi_{2} f_{2}+\Psi_{3} f_{3}
$$

In expression (11), an important role, from the point of view of the synthesis method, is played by a term that includes control:

$$
\begin{aligned}
& H^{*}=\psi_{3} * U=\left(-C_{1} * 0.2581 * e^{1.2666 t}+C_{2} *(0.1812+0.2086 i) * e^{(0.3667+1.8806 i) t}+\right. \\
& \left.+C_{3} *(0.1812-0.2086 i) * e^{(0.3667-1.8806 i) t}\right) * b U
\end{aligned}
$$

In order for the Hamiltonian $\mathrm{H}$ - expression (11) - to assume the maximum positive value, the term $\mathrm{H} *$ must always be positive and the largest. For this, the optimal control algorithm should look like:

$$
u(t)=\sigma U_{\max },
$$

where:

$$
\begin{aligned}
& \sigma=\operatorname{sign}\left(-C_{1} * 0.2581 * e^{1.2666 t}+\right. \\
& \left.\left.+C_{2} *(0.1812+0.2086 i) * e^{(0.3667+1.8806 i) t}+C_{3} *(0.1812-0.2086 i) * e^{(0.3667-1.8806 i) t}\right)\right)
\end{aligned}
$$

The optimal control $u(t)$ is a piecewise constant function taking the values $\pm U_{\max }$, and has no more than two intervals of constancy, since the nonlinear function $\psi_{3}$ changes sign not more than once. In this case, a possible change of sign occurs from plus to minus, that is, in order to fulfill the maximum principle, it is first necessary to supply $U=+U_{\max }$ to the motor, and then $U=-U_{\max }$.

With these considerations in mind, let us determine the algorithm of the optimal equation that ensures the transfer of the object from the initial state $x_{1}(0)=0, x_{2}(0)=0$, $\mathrm{x}_{3}(0)=0$ to the final state $\mathrm{x}_{1}(\mathrm{~T})=50, \mathrm{x}_{2}(\mathrm{~T})=0, \mathrm{x}_{3}(\mathrm{~T})=0$ in the minimum time $\mathrm{T}$.

It should be noted that in modern conditions there is no need to obtain an analytical solution of the original system of equations, using the available capabilities of modern software packages of the Matlab type, it is easy and straightforward to obtain a numerical solution for our case. The results of numerical simulation for the calculated optimal control algorithm are shown in Figure 1. In this case, the parameters of the model are selected, so that it fulfills the boundary condition required by the right boundary.

As can be seen from Fig. 1, the control switching time from $\left(+U_{\max }\right)$ to $\left(-U_{\max }\right)$ is selected as $t_{1}=0.5$ seconds, with the adjustment time being 1.3 seconds. The control system reaches the required output level of $50 \mathrm{rad}$., that is, the right boundary of the boundary conditions is reached. 


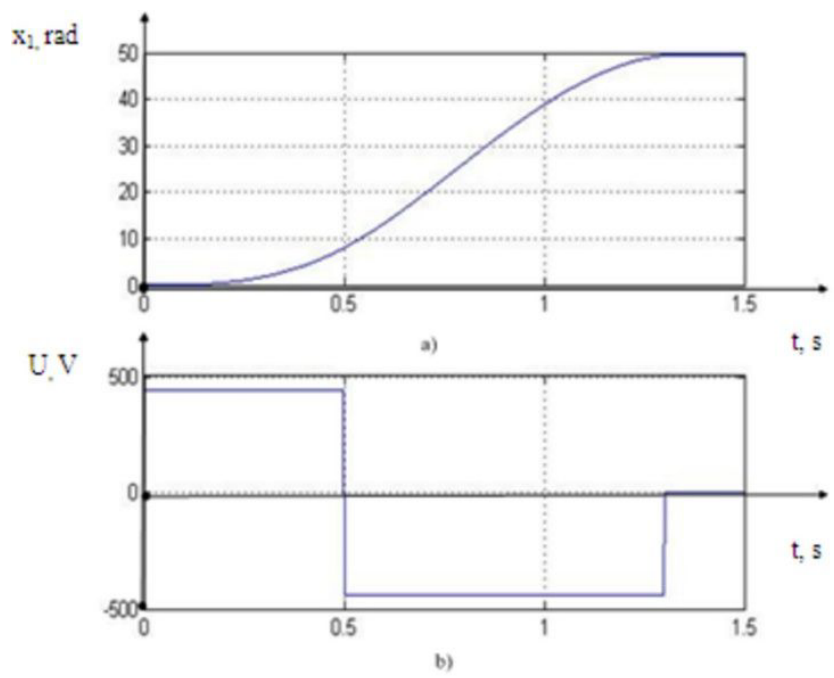

Fig. 1. Graphs: a) output coordinates of the system, b) controls

To synthesize the ACAR control system, one can not make the transition to the abstract phase case of the state space (see expression (5)), but it is convenient to use the original system (2), but it needs to be transformed to the following form:

$$
\begin{aligned}
& \frac{d x_{1}}{d t}=x_{2} \\
& \frac{d x_{2}}{d t}=-a_{21} x_{1}+a_{23} x_{3} \\
& \frac{d x_{3}}{d t}=-a_{32} x_{2}-a_{33} x_{3}+b U
\end{aligned}
$$

where the variables $\alpha=x_{1}, \omega=x_{2}, i=x_{3}$.

As permanent $\frac{c_{m}}{J}=a_{23}, \frac{C}{J}=a_{21}, \frac{c_{e}}{L}=a_{32}, \frac{R}{L}=a_{33}, \frac{1}{L}=b$ are introduced.

Taking into account the previously determined values, we obtain: $\mathrm{a}_{23}=1.515$, $\mathrm{a}_{21}=2.325, \mathrm{a}_{32}=1.5, \mathrm{a}_{33}=2, \mathrm{~b}=1$, and the system (12) takes the form:

$$
\begin{aligned}
& \frac{d x_{1}}{d t}=x_{2} \\
& \frac{d x_{2}}{d t}=-2.325 x_{1}+1.515 x_{3} \\
& \frac{d x_{3}}{d t}=-1.5 x_{2}-2 x_{3}+U
\end{aligned}
$$

Taking this into account the matrix A takes the form: $A=\left(\begin{array}{ccc}0 & 1 & 0 \\ -2.325 & 0 & 1.515 \\ 0 & -1.5 & -2\end{array}\right)$.

Knowing the matrix $\mathrm{A}$, we find the eigenvalues $\lambda$ of the matrix $\mathrm{A}$ : $\lambda_{1}=-1.2674+0.0000 \mathrm{i}, \lambda_{2}=-0.3663+1.8801 \mathrm{i}, \lambda_{3}=-0.3663-1.8801 \mathrm{i}$. As can be seen from these results, we are talking about the case of the control system considered in the synthesis by the maximum method. 
The coordinate characterizing the rotation angle of the rotor $\left(\mathrm{x}_{1}\right)$ is the output coordinate of the system; therefore, in order to form the requirements for the desired behavior of the system in the state space, we introduce the following macroscopic variable of order $\psi_{1}=\mathrm{x}_{1}-\mathrm{x}_{01} \rightarrow 0$, where $\mathrm{x}_{01}$ is the specified, required coordinate value $\mathrm{x}_{1}$. The value of the rate of change of the angle must ensure that the angle of rotation of the rotor tends to the value set above, for this we introduce another macroscopic variable $\psi_{2}=\mathrm{x}_{2}-\varphi_{2}\left(\mathrm{x}_{1}\right) \rightarrow 0$, where $\varphi_{2}\left(\mathrm{x}_{1}\right)$ is a function describing the aspiration $\mathrm{x}_{2} \rightarrow \varphi_{2}\left(\mathrm{x}_{1}\right)$ in the stationary state. Next we introduce a macroscopic order of the coordinate $\psi_{3}=x_{3}-\varphi_{3}\left(x_{1}, x_{2}\right) \rightarrow 0$, where $\varphi_{3}\left(x_{1}, x_{2}\right)$ is a function describing the relationship between the coordinates in the stationary state of the system and, $x_{3} \rightarrow \varphi_{3}\left(x_{1}, x_{2}\right)$.

For the newly introduced macro variables $\psi_{1}, \psi_{2}, \psi_{3}$, we require an asymptotically stable law of variation, that is, the fulfillment of the following system of differential equations:

$$
\left\{\begin{array}{l}
T_{1} \frac{d \Psi_{1}}{d t}+\Psi_{1}=0 \\
T_{2} \frac{d \Psi_{2}}{d t}+\Psi_{2}=0 \\
T_{3} \frac{d \Psi_{3}}{d t}+\Psi_{3}=0
\end{array}\right.
$$

where $T_{1}, T_{2}, T_{3}$ - time constants that determine the rate of aspiration of the introduced macro variables to zero, or, in other words, the rate of contraction of an arbitrary trajectory of the system's motion in the state space to the attractor required from a technological point of view.

The system of equations introduced in expression (14) expanded the state space of the system from the 3rd to the 6th, which is one of the main provisions of the ACAR method, the further synthesis of the control system is reduced to a stepwise process of decomposition (compression) of this space to an initial level [1-2]. We begin the decomposition process from the substitution in the first equation of the system (14) instead of the macro variable $\Psi 1$ its value in the coordinates of the controlled process, as a result we get:

$$
T_{1} \frac{d x_{1}}{d t}+\left(x_{1}-x_{01}\right)=0
$$

Where taking into account (13) $\frac{d x_{1}}{d t}=x_{2}$, then (15) takes the form:

$$
T_{1} x_{2}+\left(x_{1}-x_{01}\right)=0
$$

Considering the next step of the synthesis algorithm, as a result of which we assumed that $x_{2} \rightarrow \varphi_{2}\left(x_{1}\right)$, we find the value of $\varphi_{2}\left(x_{1}\right)$, as:

$$
\phi_{2}\left(x_{1}\right)=-\frac{\left(x_{1}-x_{01}\right)}{T_{1}}
$$

Taking this into account, the macro variable $\Psi_{2}$ takes the form:

$$
\Psi_{2}=x_{2}-\phi_{2}\left(x_{1}\right)=x_{2}+\frac{\left(x_{1}-x_{01}\right)}{T_{1}}
$$


The validity of expression (19), from the point of view of management objectives, confirms the fact that in the stationary state the value $\mathrm{x}_{2} \rightarrow \varphi_{2}\left(\mathrm{x}_{1}\right) \rightarrow 0$. Then, taking into account (18), the second equation of system (14) takes the following form:

$$
T_{2} \frac{d x_{2}}{d t}-T_{2} \frac{d f_{2}\left(x_{1}\right)}{d t}+x_{2}-\phi_{2}\left(x_{1}\right)=0
$$

Or taking into account $\varphi_{2}\left(x_{1}\right)$ :

$$
T_{2} \frac{d x_{2}}{d t}+\frac{T_{2}}{T_{1}} \frac{d x_{1}}{d t}+x_{2}+\frac{\left(x_{1}-x_{01}\right)}{T_{1}}=0
$$

Taking into account system (13):

$$
-T_{2} 2.325 x_{1}+T_{2} 1.515 x_{3}+\frac{T_{2}}{T_{1}} x_{2}+x_{2}+\frac{\left(x_{1}-x_{01}\right)}{T_{1}}=0
$$

In the next step of the synthesis algorithm, using the same reasoning as before and the fact that $x_{3} \rightarrow \varphi_{3}\left(x_{1}, x_{2}\right)$, we determine from (21) the value of $\varphi_{3}\left(x_{1}, x_{2}\right)$ in the coordinates of the controlled process:

$$
\phi_{3}\left(x_{1}, x_{2}\right)=1.535 x_{1}-0.66 \frac{\left(T_{2}+T_{1}\right)}{T_{1}} x_{2}-0.66 \frac{\left(x_{1}-x_{01}\right)}{T_{2} T_{1}}=0
$$

Having obtained the value of $\varphi_{3}\left(\mathrm{x}_{1}, \mathrm{x}_{2}\right)$ in the coordinates of the controlled process, we can determine the value of the macro variable $\Psi_{3}$ in the system state coordinates and open the third equation of the system (14).

$$
T_{3}\left[\frac{d x_{3}}{d t}-\frac{d \phi_{3}\left(x_{1}, x_{2}\right)}{d t}\right]+x_{3}-\phi_{3}\left(x_{1}, x_{2}\right)=0
$$

After substituting in (23) the values of $\varphi_{3}\left(\mathrm{x}_{1}, \mathrm{x}_{2}\right)$ obtained earlier from (22) and using the system (13), we obtain:

$$
\begin{aligned}
& -1.5 x_{2}-2 x_{3}+U-1.535 x_{2}-1.5 x_{1} \frac{\left(T_{1}+T_{2}\right)}{T_{1} T_{2}}+x_{3} \frac{\left(T_{1}+T_{2}\right)}{T_{1} T_{2}}+0.66 \frac{x_{2}}{T_{1} T_{2}}+\frac{x_{3}}{T_{3}}-1.535 \frac{x_{1}}{T_{3}}+\leftrightarrow \\
& \leftrightarrow 0.66 x_{2} \frac{\left(T_{1}+T_{2}\right)}{T_{1} T_{2} T_{3}}+0.66 \frac{\left(x_{1}-x_{01}\right)}{T_{1} T_{2} T_{3}}=0
\end{aligned}
$$

From the equation (24) obtained at this step of the decomposition of the state space of the control system, we define the control value in the coordinates of the controlled process:

$$
\begin{aligned}
& U=1.5 x_{2}+2 x_{3}+1.535 x_{2}+1.5 x_{1} \frac{\left(T_{1} T_{3}+T_{2} T_{3}+T_{1} T_{2}\right)}{T_{1} T_{2} T_{3}}-x_{3} \frac{\left(T_{1} T_{3}+T_{2} T_{3}+T_{1} T_{2}\right)}{T_{1} T_{2} T_{3}}-\leftrightarrow \\
& \leftrightarrow 0.66 x_{2} \frac{\left(T_{1}+T_{2}+T_{3}\right)}{T_{1} T_{2} T_{3}}-0.66 \frac{\left(x_{1}-x_{01}\right)}{T_{1} T_{2} T_{3}}
\end{aligned}
$$

Expression (25) determines an asymptotically stable control action on the control system described by equations (13).

To limit the maximum control value, we introduce into the control loop a non-linear link of the "saturation" type with the same threshold as in the case of a maximum. The results of the system simulation taking into account this threshold and with the selected values of the constants $T_{1}, T_{2}, T_{3}$ are shown in Fig. 2. 


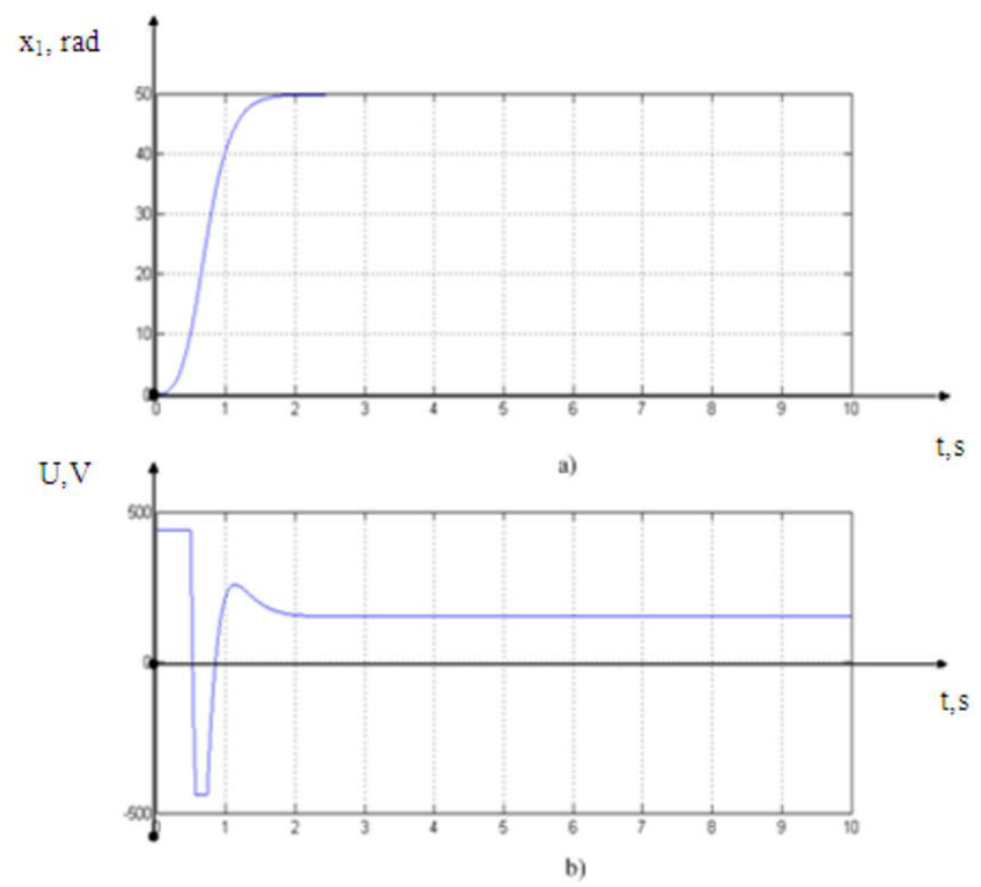

Fig. 2. Results of modeling with control at $\mathrm{T} 1=0.15, \mathrm{~T} 2=0.16, \mathrm{~T} 3=0.17$,

a) the transient process on the output coordinate, b) the control

As can be seen from Figure 2, the introduction of a non-linear constraint on control of the "saturation" type makes it possible to obtain the result required from the point of view of the admissibility of control, while the control structure defined by the maximum method is actually observed, but control is not optimal in speed.

\section{Discussion of results}

The laws synthesized in the work showed a similar control structure, but the control synthesized by the ACAR method does not allow the control system to solve the task for as short a time as in the case of the maximum principle. However, the time constants $T_{1}, T_{2}$, $\mathrm{T}_{3}$ introduced in the ACAR synthesis play a role similar to the role that is determined by the switching time of the control in the case of the control implementation based on the maximum principle. Proceeding from these considerations, the following hypothesis is suggested: when comparing the control obtained by the ACAR method and control on the basis of the maximum principle, taking into account the introduction of an additional nonlinear element that limits the amplitude of the control signal, the selection of the introduced time constants $\mathrm{T}_{1}, \mathrm{~T}_{2}, \mathrm{~T}_{3}$ allows to obtain the speed of this system close to the optimal one, which is computed for the case of realization of the system on the basis of the maximum principle. Thus, the proposed approach makes it possible to obtain a quasioptimal control system synthesized by the ACAR method.

\section{References}

1. L.S. Pontryagin, V.G. Boltyanskii, et al., Mathematical Theory of Optimal Processes, (Nauka, Moscow, 1983) 
2. A.A. Kolesnikov, Applied synergetics: fundamentals of system synthesis (Publishing house of TIT SFedU, Taganrog, 2007)

3. A.A. Kolesnikov, Synergetics and problems of control theory (Fizmatlit, Moscow, 2004)

4. O.V. Uzunov, Solid State Phenomena, 147-149, 468 (2009)

5. V.L. Zakavorotny, V.P. Lapshin, and A.A. Gubanova, Izvestiya Vysshikh Uchebnykh Zavedenii. North-Caucasian region (Engineering), 4, 59 (2014)

6. VL Zakborotny, I.A. Turkin, V.P. Lapshin, Izvestiya Vysshikh Uchebnykh Zavedenii. North-Caucasian region (Engineering), 3, 128 (2014)

7. V.P. Lapshin, and I.A. Turkin, Bulletin of the Adyghe State University (NaturalMathematical and Technical Sciences), 4, 226 (2012)

8. V.P. Lapshin, I.A. Turkin, Russian Engineering Research, 35, 10, 795 (2015)

9. V.P. Lapshin, I.A. Turkin, Procedia Engineering, 206, 594 (2017)

10. V.L. Zakovorotny, V.P. Lapshin, T.S. Babenko, Procedia Engineering, 206, 68 (2017)

11. V.L. Zakavorotny, V.P. Lapshin, I.A. Turkin, Izvestiya Vysshikh Uchebnykh Zavedenii. North-Caucasian region (Engineering), 1, 36 (2014)

12. V.L. Zakavorotny, V.P. Lapshin, and I.A. Turkin, Izvestiya Vysshikh Uchebnykh Zavedenii. North-Caucasian region (Engineering), 3, 33 (2014)

13. V.P. Lapshin, I.A. Turkin, Automotive industry, 1, 16 (2017)

14. A.G. Mladov, M. Vysshaya shkola, 223 (1966) 